\title{
Efficacy and safety of intratumoral thermotherapy using magnetic iron-oxide nanoparticles combined with external beam radiotherapy on patients with recurrent glioblastoma multiforme
}

\author{
Klaus Maier-Hauff • Frank Ulrich • Dirk Nestler • \\ Hendrik Niehoff · Peter Wust · Burghard Thiesen · \\ Helmut Orawa $\cdot$ Volker Budach $\cdot$ Andreas Jordan
}

Received: 11 June 2010/Accepted: 26 August 2010/Published online: 16 September 2010

(C) The Author(s) 2010. This article is published with open access at Springerlink.com

\begin{abstract}
Therapy options at the time of recurrence of glioblastoma multiforme are often limited. We investigated whether treatment with a new intratumoral thermotherapy procedure using magnetic nanoparticles improves survival outcome. In a single-arm study in two centers, 66 patients (59 with recurrent glioblastoma) received neuronavigationally controlled intratumoral instillation of an aqueous dispersion of iron-oxide (magnetite) nanoparticles and subsequent heating of the particles in an alternating magnetic field. Treatment was combined with fractionated stereotactic radiotherapy. A median dose of 30 Gy using a fractionation of $5 \times 2 \mathrm{~Gy} /$ week was applied. The primary study endpoint was overall survival following diagnosis of first tumor recurrence (OS-2), while the secondary endpoint was overall survival after primary tumor diagnosis (OS-1). Survival times were calculated using the Kaplan-Meier
\end{abstract}

Disclosure Andreas Jordan and Burghard Thiesen are employees of MagForce Nanotechnologies.

\author{
K. Maier-Hauff $(\bowtie) \cdot$ D. Nestler \\ Department of Neurosurgery, Bundeswehrkrankenhaus Berlin, \\ Scharnhorststr. 13, 10115 Berlin, Germany \\ e-mail: maier-hauff@cbn-berlin.de \\ F. Ulrich · H. Niehoff \\ Clinic for Neurosurgery, Helios Klinikum Krefeld, \\ Krefeld, Germany \\ P. Wust · V. Budach · A. Jordan \\ Department of Radiotherapy, Charité-Universitätsmedizin \\ Berlin, Berlin, Germany \\ B. Thiesen · A. Jordan \\ MagForce Nanotechnologies AG, Berlin, Germany \\ H. Orawa \\ Coordination Centre for Clinical Studies (KKS), \\ Charité-Universitätsmedizin Berlin, Berlin, Germany
}

method. Analyses were by intention to treat. The median overall survival from diagnosis of the first tumor recurrence among the 59 patients with recurrent glioblastoma was 13.4 months (95\% CI: 10.6-16.2 months). Median OS-1 was 23.2 months while the median time interval between primary diagnosis and first tumor recurrence was 8.0 months. Only tumor volume at study entry was significantly correlated with ensuing survival $(P<0.01)$. No other variables predicting longer survival could be determined. The side effects of the new therapeutic approach were moderate, and no serious complications were observed. Thermotherapy using magnetic nanoparticles in conjunction with a reduced radiation dose is safe and effective and leads to longer OS-2 compared to conventional therapies in the treatment of recurrent glioblastoma.

Keywords Glioblastoma - Magnetic nanoparticles · Radiotherapy · Recurrence $\cdot$ Thermotherapy

\section{Introduction}

For most patients with newly diagnosed glioblastoma multiforme (GBM), tumor resection followed by postoperative radiotherapy combined with temozolomide has become the standard of care $[1,2]$. However, when the tumor recurs, subsequent treatment becomes highly casespecific, taking into account the treatment history along with the size and location of the tumor. Not all patients are eligible for every type of salvage therapy, but a subset of patients may benefit from maximal treatment efforts [3].

Surgical removal of the recurrent tumor should be considered in all patients [4]. Repeated resection of recurrent GBM has shown improvement in progression-free survival [5], though its general efficacy and utility clearly depends 
on the expertise and attitude of the respective surgeon. Re-irradiation is possible in many patients [6-11], and the use of stereotactic techniques in particular has resulted in an increase in post-recurrence median survival to 8-10 months [12-14]. Nevertheless, there is still no general agreement on which radiation technique and fractionation is most appropriate, and the choice often seems to depend upon the radiotherapy options available to the treating physician. Chemotherapy is the most common treatment option for recurrent glioblastoma, and numerous studies have demonstrated the safety and efficacy of various agents, both alone and in combination [15-19].

In addition to these standard therapies, alternative therapeutic approaches have to some extent produced promising results or are the subject of ongoing investigations [20-25]. The need for more efficacious treatment options, however, remains great. One of these, hyperthermia, has already demonstrated efficacy specifically in the treatment of glioblastoma [26], but the high surgical effort required to implant catheter arrays within the tumor along with the non-negligible patient morbidity have hindered its establishment in clinical practice.

The combination of hyperthermia with radiotherapy increases cytotoxic effects, and several clinical trials investigating different techniques in different tumor sites have shown benefits from combined treatment [27-29]. In our new approach, namely intratumoral thermotherapy using magnetic nanoparticles (Nano-Cancer ${ }^{\circledR}$ therapy), biocompatible iron-oxide nanoparticles are directly injected into the tumor and subsequently stimulated by an alternating magnetic field to generate heat. The feasibility and efficacy of this approach were demonstrated in preclinical [30, 31], clinical [32-34] and post-mortem studies [35].

The objective of the present study was to determine the efficacy of intratumoral thermotherapy in conjunction with fractionated stereotactic radiotherapy for the treatment of recurrent glioblastoma.

\section{Methods}

Study design and patients

This prospective, single-arm, two-center phase II study was initiated in April 2005 and closed to accrual in September 2009. The study population consisted of 66 patients. Seven of these did not fulfill the inclusion criteria, such that a total of 59 patients ( $54 \%$ male) with recurrent glioblastoma were included in the statistical analysis of overall survival.

The key criteria for eligibility were: histologic diagnosis of supratentorial glioblastoma; unequivocal evidence of recurrence or progression (as determined by the referring physicians using MRI) with up to three additional foci; maximum tumor dimension of $7 \mathrm{~cm}$; absence of tumor infiltration into the ventricle, brain stem, corpus callosum or contralateral site; age between 18 and 75 years; Karnofsky performance score (KPS) $\geq 60$; and a life expectancy of at least 3 months. Irremovable metallic parts in the treatment area, cardiac pacemaker and implanted defibrillator were exclusion criteria. The study, approved by the institutional review boards of the participating centers, was conducted according to the principles of the Declaration of Helsinki. All patients certified their fully informed consent.

Following the treatment procedure, patients were monitored at 3-month intervals with follow-up clinical examinations including CT scans. One patient was lost during the follow-up period, and in this case survival was deemed until the time of the last CT scan. Data on any subsequent treatments for tumor progression following the thermo-/ radiotherapy were not systematically collected.

Magnetic field applicator and magnetic fluid

The thermotherapy was performed using the alternating magnetic field applicator MFH 300F with integrated thermometry unit (NanoActivator ${ }^{\circledR}$ F100; MagForce Nanotechnologies, Berlin, Germany). The strength of the alternating $(100 \mathrm{kHz})$ magnetic field can be adjusted from 2 to $15 \mathrm{kA} / \mathrm{m}$. The applicator is designed for universal usage in treating tumors anywhere in the body.

The magnetic fluid MFL AS1 (NanoTherm ${ }^{\circledR}$ AS1; MagForce Nanotechnologies), an aqueous dispersion of superparamagnetic nanoparticles with an iron concentration of $112 \mathrm{mg} / \mathrm{ml}$, served as the energy transducer. The nanoparticles are formed as iron-oxide magnetite $\left(\mathrm{Fe}_{3} \mathrm{O}_{4}\right)$ cores of approx. $12 \mathrm{~nm}$ diameter with an aminosilane coating, which acts to ensure that the nanoparticle deposits remain stable within the tumor tissue. The magnetite cores possess an intrinsic magnetic moment, which can be stimulated by the externally applied alternating magnetic field to create heat through relaxation processes. The high concentration of iron was necessary to generate sufficient heat within the tumor for effective thermotherapy, while simultaneously minimizing the volume of instilled fluid.

Instillation and thermotherapy

Prior to treatment, it was first necessary to remove all metallic materials within $40 \mathrm{~cm}$ of the treatment area, particularly dental fillings, crowns and implants.

The magnetic fluid was instilled using neuronavigational control under general anesthesia in a procedure comparable to a brain needle biopsy. The median amount of magnetic fluid injected was $4.5 \mathrm{ml}$ (range $0.5-11.6 \mathrm{ml}$ ), corresponding to a median dosage of $0.28 \mathrm{ml}$ of magnetic fluid per $\mathrm{cm}^{3}$ of tumor volume. Following instillation of the 
magnetic fluid, a closed-end thermometry catheter (outside diameter $1.0 \mathrm{~mm}$ ) was placed in the target area.

Thereafter, the nanoparticle density was mapped using CT imaging so that the treatment parameters for the subsequent intratumoral thermotherapy could be planned (Fig. 1). On the basis of the density distribution of the nanoparticles, their known specific absorption rate (SAR), and the estimated perfusion within the tumor area, the heat generation within the target tissue could be determined as a function of magnetic field strength using the bioheat transfer equation. The aim was to establish the magnetic field strength such that a temperature of $43^{\circ} \mathrm{C}$ was not exceeded beyond a margin of $2 \mathrm{~cm}$ around the tumor. Further details of this treatment planning process, referred to as post-instillation analysis (PIA), have been described by Gneveckow et al. [36].
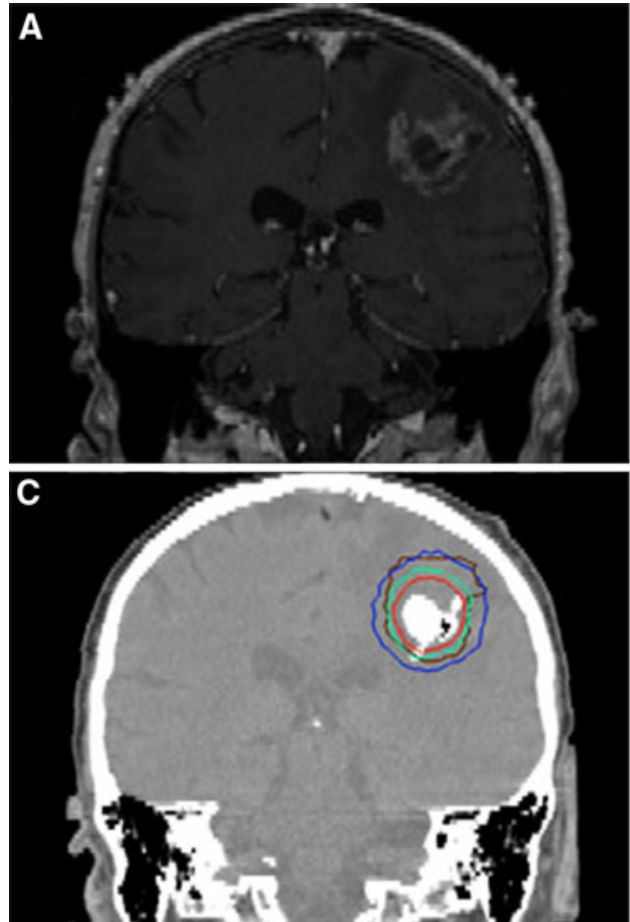

E

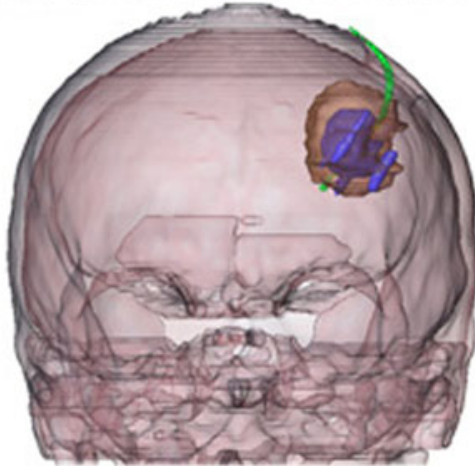

Fig. 1 Glioblastoma recurrence. a,b Pre-treatment brain MRI. c,d Post-instillation CT showing magnetic nanoparticle deposits as hyperdense areas. Isothermal lines indicate calculated treatment temperatures between $40^{\circ} \mathrm{C}$ (blue) and $50^{\circ} \mathrm{C}$ (red). The brown line
The hyperthermia treatment generally consisted of six semi-weekly sessions, and each thermotherapy session lasted $1 \mathrm{~h}$. During the first two sessions, the procedure was monitored using direct temperature measurements from the previously placed thermometry catheter. Since the maximum field strength of the remaining sessions did not exceed that of the first two sessions, these were performed without direct temperature measurement. The median peak temperature measured within the tumor area during the thermotherapy sessions was $51.2^{\circ} \mathrm{C}$ (maximum $82.0^{\circ} \mathrm{C}$ ).

\section{Adjunct radiotherapy}

Stereotactic beam radiotherapy was performed immediately before or after the intratumoral thermotherapy sessions with a $6 \mathrm{MV}$ Novalis system (Varian, Palo Alto, USA/
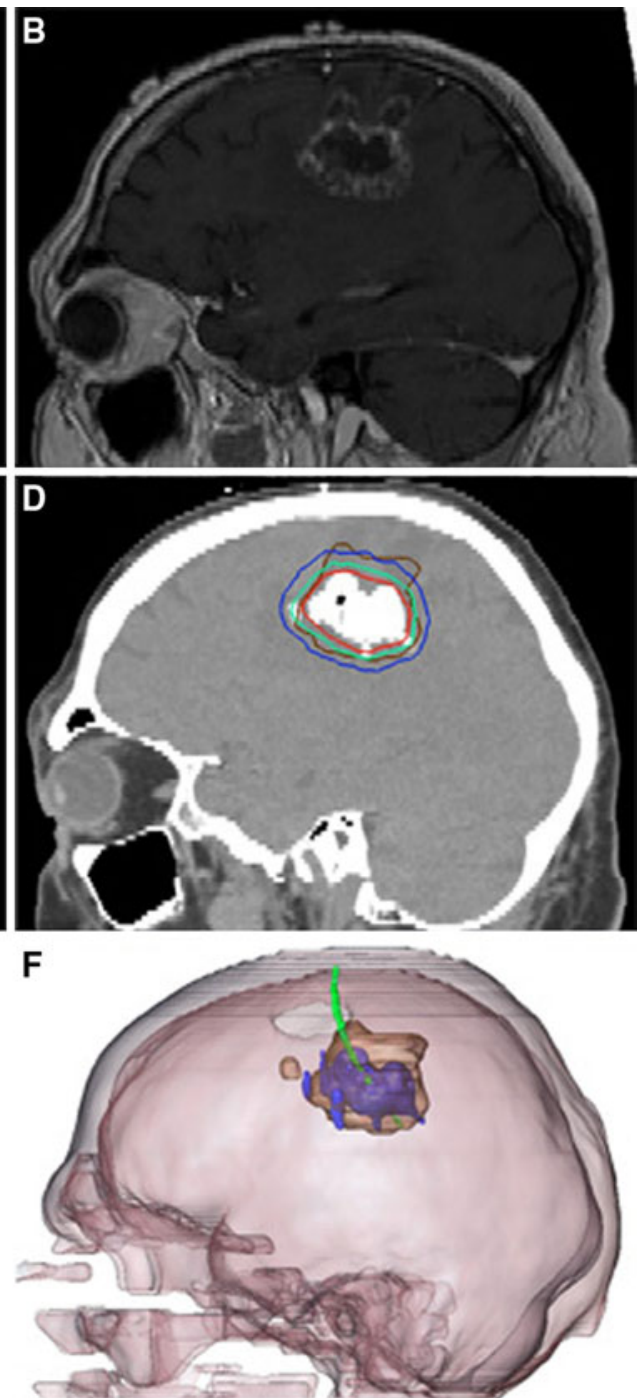

represents the tumor area. e,f 3-D reconstruction of fused MRI and CT showing the tumor (brown), magnetic fluid (blue) and thermometry catheter (green) 
BrainLAB, Kirchheim, Germany). The planning target volume (PTV) enclosed the regions of contrast enhancement including any resection cavity and an additional margin of $3 \mathrm{~mm}$. The median PTV was $46.5 \mathrm{~cm}^{3}$ (range $6.6-108.0 \mathrm{~cm}^{3}$ ). Treatment planning for radiotherapy was done using Brainscan ${ }^{\circledR}$ or iPlan ${ }^{\circledR}$ software (BrainLAB, Feldkirchen, Germany). A biologically equivalent median dose (BED) of $30 \mathrm{~Gy}$ was administered in $\geq 95 \%$ of the PTV, generally fractionated as $5 \times 2$ Gy per week.

\section{Statistical analysis}

Survival times were calculated using the Kaplan-Meier method. The primary endpoint of the study was overall survival following the first diagnosis of tumor recurrence or progression (OS-2). The secondary endpoint was overall survival from the time point of primary diagnosis of glioblastoma (OS-1). Influence of prognostic factors on survival was evaluated using the logrank test and correlation analysis was done using Pearson's correlation coefficient. The software program used for the statistical analyses was SPSS Statistics 18.

\section{Results}

Median patient age at study entry was 55.7 years. A total of 41 patients had previously received a complete and 15 a subtotal tumor resection upon primary diagnosis. In 2 patients, only a biopsy had been performed, and in 1 patient the extent of resection could not be retraced. The median time interval between diagnosis of primary glioblastoma and first tumor recurrence/progression (TTP-1) was 8.0 months. Further details of the study population are listed in Table 1 .

Table 1 Patient characteristics $(n=59)$

\begin{tabular}{lll}
\hline & No. & $(\%)$ \\
\hline First-line therapy & & \\
Resection & 56 & 95 \\
Radiotherapy & 58 & 98 \\
Chemotherapy & 51 & 86 \\
Patients with prior treatment following & 24 & 41 \\
tumor recurrence but before study entry & & \\
Resection & 11 & 19 \\
Radiotherapy & 2 & 3 \\
Chemotherapy & 17 & 29 \\
KPS at study entry—median (range) & $90(60-100)$ \\
Karnofsky performance score (KPS) $\geq 80$ & 46 & 78 \\
Age in years at study entry-median & 55.7 & \\
Patients with age $<50$ & 23 & 39 \\
Patients with age $\geq 50$ & 36 & 61 \\
\hline
\end{tabular}

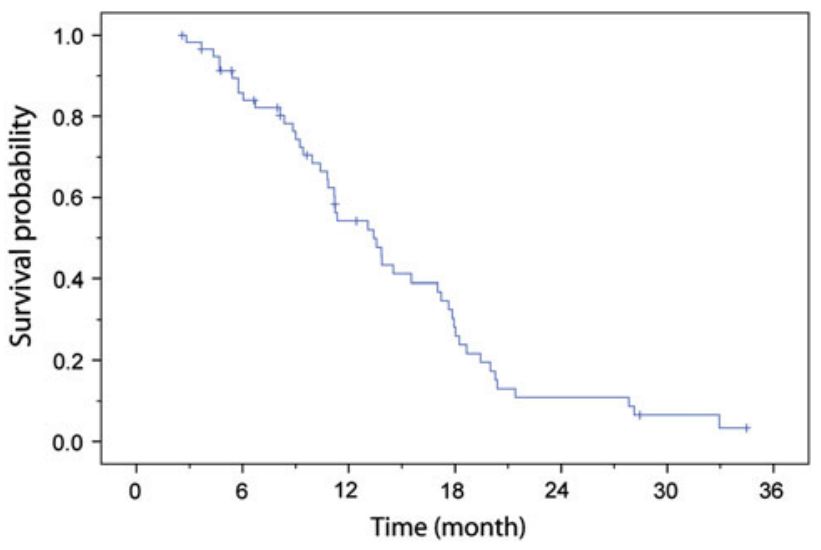

Fig. 2 Overall survival after diagnosis of first tumor recurrence/ progression (OS-2) of 59 glioblastoma patients treated with combined thermo-/radiotherapy for reintervention

Over the study period ending 30 September 2009, 47 out of the 59 patients with recurrent glioblastoma died. In only 41 of these was there no doubt that death was caused by tumor growth. However, in order to be conservative, all 47 deaths were regarded as tumor-related.

Median OS-2 estimated using the Kaplan-Meier method was 13.4 months (95\% CI: 10.6-16.2 months) in the study population (Fig. 2). In differentiating the study population between those who had had prior treatment following the first tumor recurrence $(n=24$, see Table 1) and those who had not $(n=35)$, the median OS-2 for those who had was 13.9 months (95\% CI: 10.9-16.8), compared to 11.2 months (95\% CI: 6.7-15.6) for those who had not. Median OS-1 was 23.2 months with a $95 \%$ confidence interval of 17.2-29.2 months.

All standard prognostic factors were analyzed for their impact on survival. Only tumor volume at study entry was significantly correlated with ensuing survival (log-rank test, $P<0.01)$. No other factors-age, KPS, TTP-1, or prior treatment following first recurrence-had any significant prognostic value on OS-2. Only between TTP-1 and OS- 1 was there a positive linear correlation which was significantly different from zero $(r=0.65 ; P<0.01)$. The Pearson's correlation coefficient $(r)$ between TTP-1 and OS-2, however, was almost zero (no relationship). No correlations were observed between tumor location, size or attained treatment temperatures and either OS-2 or observed side effects.

Acute side effects during thermotherapy were classified according to version 2.0 of the Common Toxicity Criteria (CTC). The following side effects were observed in more than one patient.

During thermotherapy sessions, 33 patients $(50.0 \%)$ exhibited sweating up to grade 1 , and $31(47.0 \%)$ reported a general sensation of warmth in the treatment area. In 6 patients $(9.1 \%)$, body temperature during thermotherapy exceeded $38^{\circ} \mathrm{C}$ (grade $1-3$ thermal stress), while the body 
temperature of all other patients increased only slightly during the treatment procedure. No correlation could be established between body temperature elevation and the size or number of lesions being treated.

Twelve patients (18.2\%) demonstrated grade 1 tachycardia during the thermotherapy procedure. Short-term fluctuations in blood pressure not requiring treatment were observed in 10 patients: hypertonia in $7(10.6 \%)$, and hypotonia in $3(4.5 \%)$.

Nine patients (13.6\%) reported headaches during thermotherapy. These were generally mild and temporary, but in 3 patients they led to individual thermotherapy sessions being aborted. It is possible that they were caused by a transitory increase in intracranial pressure resulting from the local increase in temperature. Fifteen patients (22.7\%) experienced convulsions which were mostly focal. Anti-epileptic drugs (AEDs) already being taken by patients were maintained. While AEDs were not administered prophylactically prior to commencement of therapy in this study, this approach should be considered in the future. Fourteen patients (21.2\%) exhibited motor disturbances, mostly as a worsening of already existing hemiparesis. In 4 of these, the symptoms appeared following the nanoparticle instillation procedure, while in the others, motor disturbances were observed after a varying number of thermotherapy sessions. By using CT imaging, an enlargement of perifocal edema could be observed in 6 of these patients. However, only $2(3.0 \%)$ of the patients experiencing convulsions or motor disturbances demonstrated such neurological disorders for the first time.

Except for worsening of hemiparesis, no prolonged side effects were observed. In 19 patients, key parameters of iron metabolism (ferritin, transferrin, transferrin saturation, serum iron) were tested both before and after the instillation of magnetic fluid, and there was no indication of iron being released from the intratumoral deposits or being metabolized.

\section{Discussion}

The objective of the present single-arm study was to demonstrate the clinical efficacy of the newly developed intratumoral thermotherapy using magnetic nanoparticles in conjunction with percutaneous irradiation for the treatment of recurrent GBM.

Because it is not randomized, this study specifically recognizes the potential for selection bias. A randomization would have been possible in theory but extremely difficult in practice because patients with recurrent glioblastoma are acutely aware of their impending mortality and therefore typically make a highly considered decision about any participation in trials of a new experimental therapy. Most would be loath to participate in a randomized study where they might only be receiving supportive treatment, and thus recruiting patients for randomized trials would have been difficult and considerably extended the time required for this study.

As with many other carcinomas, pre-therapeutic prognostic factors are major determinants of overall survival $[37,38]$, and survival may show greater correlation to these factors than any specific treatment. Selecting historical controls from past studies is thus not unproblematic since these frequently involve only small populations with differing prognostic factors (particularly KPS, age and prior treatment). For this reason, many studies have utilized the metaanalysis by Wong et al. [39] as a baseline historical reference population. According to their study, the median survival for patients treated with chemotherapy following glioblastoma recurrence was 5.8 months. However, the recently published results of the EORTC-NCIC trial on primary glioblastoma by Stupp et al. [1,2] reflect the more recent use of temozolomide as the current standard in the treatment of primary glioblastoma, and thus its results establish, in principle, a new baseline for survival data. Its OS-2 is particularly well suited to direct comparison with future study results because the primary therapy was conducted on a large patient population $(n=287)$ using this currently prevailing standard treatment, while upon recurrence/progression, patients were treated at the local investigators' discretion. The OS-2 data from this study may therefore be viewed as broadly representative of median survival using the range of salvage therapies currently available.

While recognizing the issues of data comparability between our's and other studies, the extension of median OS-2 to 13.4 months in our study compared to 6.2 months in the Stupp study population [2] can clearly be regarded as significant (using the statistical method of Simon [40]). It should specifically be noted that it is not the results of the primary therapy being compared but rather survival following the best possible treatment upon tumor recurrence after standard first-line treatment.

Further evidence suggesting superiority of this new therapy is provided by the median secondary study endpoint OS-1, which at 23.2 months substantially exceeded the median 14.6 months in the reference group [1]. In comparing the increase in median OS-2 of 7.2 months to the gain in OS- 1 of 8.6 months, it is apparent that the great majority of the gain in overall survival followed recurrence and thus can be specifically attributed to the thermo-/ radiotherapy. If one restricts the study group to only those patients who had not received any prior treatment following their first recurrence $(n=35)$, the median OS-2 for this subset is shortened to 11.2 months but still much greater than 6.2 months. It may thus be inferred that the observed increase in survival is not attributable to any prior treatment of the recurrence before study entry. The remaining patients who had been previously treated $(n=24)$ had a median OS-2 of 13.9 months. 
The median age of the study population (56 years) was extremely close to the reference population (57 years), and median TTP-1 differed only modestly (8.0 vs 6.9 months), suggesting that the study results were not materially affected by patient selection and thus that they are clinically relevant. Data on other prognostic factors, such as tumor volume and KPS at recurrence, were unfortunately not available for the reference population.

In addition to examining the potential role of patient selection on survival, the extent to which the adjunct radiotherapy might have contributed to the increased survival must also be addressed. Prior studies of stereotactic fractionated radiotherapy following GBM recurrence, however, demonstrate only a lower increase in median survival, and in small patient groups. Hudes et al. [8] and Vordermark et al. [13] attained respective OS-2 of 10.5 months $(n=19)$ and 7.9 months $(n=14)$ using hypofractionated stereotactic radiotherapy, but both these studies involved relatively small tumor volumes. However, a retrospective study by Combs et al. involving similar tumor volumes in a comparable patient population (in terms of KPS and age) found a median survival of 8.1 months following fractionated stereotactic re-irradiation at a level of 36 Gy [12].

The remarkable increase in overall survival in our study through the combination of thermotherapy with a lower radiotherapy dosage of $30 \mathrm{~Gy}$ thus indicates the efficacy of the applied heat, at least in this combination. It must be underscored that the studies of stereotactic radiosurgery involving tumors of significantly smaller volume do not necessarily offer a valid basis of comparison with our study results.

Even with deliberate selection of patients being treated for recurrent GBM using predefined criteria, Hau et al. [3] could demonstrate only a modest increase in OS-2 to 7.6 months, although it must be noted that, with a median KPS of 70 and a TPP- 1 of 6 months, the prognosis for their patient group was presumably slightly worse than for our own study population.

With regard to safety, intratumoral thermotherapy offers an approach to hyperthermia (or thermoablation) with only moderate side effects.

Two drawbacks of this new approach which warrant mention are, firstly, the need to remove all metal from within $40 \mathrm{~cm}$ of the treatment area (i.e., all dental work), and secondly, the indefinite exclusion of MRI for subsequent diagnosis of tumor progression. There remain, however, other effective methods beside CT to monitor disease progression including PET and SPECT, which can be regarded at least as good as MRI. Outside the area of nanoparticle deposits, conventional MRI according to current practice may still be used without any limitation. It should be noted that the exclusion of MRI for evaluating tumor progression arises not from the effects of MRI on the magnetic nanoparticles but, conversely, from the MRI artifacts resulting from the very high particle concentrations used. By comparison, iron-oxide nanoparticles are used in much lower concentrations for therapeutic targeting and MRI contrast enhancement of glioblastoma [41].

In looking at the entirety of the observed side effects, intratumoral thermotherapy may be regarded as safe and well tolerated compared to other available treatment options. This study likewise demonstrates that, in the indicated patient group, this new therapeutic approach in conjunction with fractionated stereotactic radiotherapy is clinically effective. The increase in overall survival points to a clearly favorable risk-benefit ratio in patients with recurrent GBM.

Intratumoral thermotherapy may, in principle, be used in combination with any conventional therapy in order to amplify its effects and thus offers the potential for even greater potency. Because of the stability of the nanoparticle deposits, and in contrast to radiotherapy, which is subject to cumulative dosage limits, the thermotherapy sessions may be repeated or combined with other therapies without any inherent limit. The combination of intratumoral hyperthermia and chemotherapy (particularly temozolomide) presents a particularly promising approach which warrants further clinical investigation. The delivery of nanoparticles into the tumor using convection-enhanced delivery (CED) might be a potential alternative to direct intratumoral instillation and is thus also a potential subject for examination in future clinical studies.

Furthermore, because there are only marginal differences in temperature sensitivity between different body tissues [27], one may suppose that patients with other solid tumors besides glioblastoma might benefit from this novel approach provided that sufficient temperatures can be safely attained in these tumors. This is the subject of other clinical studies currently in progress.

Acknowledgments The study was funded by MagForce Nanotechnologies. We thank Heike Bender for study management, JensThorsten Ollek for conducting the post-instillation analyses, Norbert Waldöfner for synthesizing the magnetic fluid, and Regina Scholz for her helpful suggestions as to intracranial nanoparticle application.

\section{Conflict of interest None.}

Open Access This article is distributed under the terms of the Creative Commons Attribution Noncommercial License which permits any noncommercial use, distribution, and reproduction in any medium, provided the original author(s) and source are credited.

\section{References}

1. Stupp R, Mason WP, van den Bent MJ et al (2005) Radiotherapy plus concomitant and adjuvant temozolomide for glioblastoma. N Engl J Med 352:987-996 
2. Stupp R, Hegi ME, Mason WP et al (2009) Effects of radiotherapy with concomitant and adjuvant temozolomide versus radiotherapy alone on survival in glioblastoma in a randomised phase III study: 5-year analysis of the EORTC-NCIC trial. Lancet Oncol 10:459-466

3. Hau P, Baumgart U, Pfeifer K et al (2003) Salvage therapy in patients with glioblastoma: is there any benefit? Cancer 98:2678-2686

4. Sawaya R (1999) Extent of resection in malignant gliomas: a critical summary. J Neurooncol 42:303-305

5. Stummer W, Pichlmeier U, Meinel T, Wiestler OD, Zanella F, Reulen HJ (2006) Fluorescence-guided surgery with 5-aminolevulinic acid for resection of malignant glioma: a randomised controlled multicentre phase III trial. Lancet Oncol 7:392-401

6. Nieder C, Astner ST, Mehta MP, Grosu AL, Molls M (2008) Improvement, clinical course, and quality of life after palliative radiotherapy for recurrent glioblastoma. Am J Clin Oncol 31:300-305

7. Cho KH, Hall WA, Gerbi BJ, Higgins PD, McGuire WA, Clark HB (1999) Single dose versus fractionated stereotactic radiotherapy for recurrent high-grade gliomas. Int J Radiat Oncol Biol Phys 45:1133-1141

8. Hudes RS, Corn BW, Werner-Wasik M et al (1999) A phase I dose escalation study of hypofractionated stereotactic radiotherapy as salvage therapy for persistent or recurrent malignant glioma. Int J Radiat Oncol Biol Phys 43:293-298

9. Larson DA, Suplica JM, Chang SM et al (2004) Permanent iodine 125 brachytherapy in patients with progressive or recurrent glioblastoma multiforme. Neuro-oncol 6:119-126

10. Simon JM, Cornu P, Boisserie G et al (2002) Brachytherapy of glioblastoma recurring in previously irradiated territory: predictive value of tumor volume. Int J Radiat Oncol Biol Phys 53:67-74

11. Chan TA, Weingart JD, Parisi M et al (2005) Treatment of recurrent glioblastoma multiforme with GliaSite brachytherapy. Int J Radiat Oncol Biol Phys 62:1133-1139

12. Combs SE, Gutwein S, Thilmann C, Huber P, Debus J, SchulzErtner D (2005) Stereotactically guided fractionated re-irradiation in recurrent glioblastoma multiforme. J Neurooncol 74:167-171

13. Vordermark D, Kolbl O, Ruprecht K, Vince GH, Bratengeier K, Flentje M (2005) Hypofractionated stereotactic re-irradiation: treatment option in recurrent malignant glioma. BMC Cancer 5:55

14. Shrieve DC, Alexander E 3rd, Wen PY et al (1995) Comparison of stereotactic radiosurgery and brachytherapy in the treatment of recurrent glioblastoma multiforme. Neurosurgery 36:275-282 discussion 274-282

15. Gwak HS, Youn SM, Kwon AH, Lee SH, Kim JH, Rhee $\mathrm{CH}$ (2005) ACNU-cisplatin continuous infusion chemotherapy as salvage therapy for recurrent glioblastomas: phase II study. J Neurooncol 75:173-180

16. Brandes AA, Tosoni A, Franceschi E et al (2009) Fotemustine as second-line treatment for recurrent or progressive glioblastoma after concomitant and/or adjuvant temozolomide: a phase II trial of Gruppo Italiano Cooperativo di Neuro-Oncologia (GICNO). Cancer Chemother Pharmacol 64:769-775

17. Keles GE, Lamborn KR, Chang SM, Prados MD, Berger MS (2004) Volume of residual disease as a predictor of outcome in adult patients with recurrent supratentorial glioblastomas multiforme who are undergoing chemotherapy. J Neurosurg 100:41-46

18. Wick A, Felsberg J, Steinbach JP et al (2007) Efficacy and tolerability of temozolomide in an alternating weekly regimen in patients with recurrent glioma. J Clin Oncol 25:3357-3361

19. Brandes AA, Tosoni A, Cavallo G et al (2006) Temozolomide 3 weeks on and 1 week off as first-line therapy for recurrent glioblastoma: phase II study from gruppo italiano cooperativo di neuro-oncologia (GICNO). Br J Cancer 95:1155-1160
20. Buie LW, Valgus J (2008) Bevacizumab: a treatment option for recurrent glioblastoma multiforme. Ann Pharmacother 42:1486-1490

21. Rich JN, Reardon DA, Peery T et al (2004) Phase II trial of gefitinib in recurrent glioblastoma. J Clin Oncol 22:133-142

22. Schwarzmaier HJ, Eickmeyer F, von Tempelhoff W et al (2006) MR-guided laser-induced interstitial thermotherapy of recurrent glioblastoma multiforme: preliminary results in 16 patients. Eur $\mathbf{J}$ Radiol 59:208-215

23. Gutin PH, Iwamoto FM, Beal K et al (2009) Safety and efficacy of bevacizumab with hypofractionated stereotactic irradiation for recurrent malignant gliomas. Int J Radiat Oncol Biol Phys 75:156-163

24. Darakchiev BJ, Albright RE, Breneman JC, Warnick RE (2008) Safety and efficacy of permanent iodine- 125 seed implants and carmustine wafers in patients with recurrent glioblastoma multiforme. J Neurosurg 108:236-242

25. Boiardi A, Silvani A, Eoli M et al (2008) Treatment of recurrent glioblastoma: can local delivery of mitoxantrone improve survival? J Neurooncol 88:105-113

26. Sneed PK, Stauffer PR, McDermott MW et al (1998) Survival benefit of hyperthermia in a prospective randomized trial of brachytherapy boost \pm hyperthermia for glioblastoma multiforme. Int J Radiat Oncol Biol Phys 40:287-295

27. Wust P, Hildebrandt B, Sreenivasa G et al (2002) Hyperthermia in combined treatment of cancer. Lancet Oncol 3:487-497

28. van der Zee J, Gonzalez Gonzalez D, van Rhoon GC, van Dijk JD, van Putten WL, Hart AA (2000) Comparison of radiotherapy alone with radiotherapy plus hyperthermia in locally advanced pelvic tumours: a prospective, randomised, multicentre trial. Dutch Deep Hyperthermia Group. Lancet 355:1119-1125

29. Issels RD, Lindner LH, Verweij J, Wust P (2010) Neo-adjuvant chemotherapy alone or with regional hyperthermia for localised high-risk soft-tissue sarcoma: a randomised phase 3 multicentre study. Lancet Oncol 11:561-570

30. Jordan A, Scholz R, Maier-Hauff K et al (2006) The effect of thermotherapy using magnetic nanoparticles on rat malignant glioma. J Neurooncol 78:7-14

31. Johannsen M, Thiesen B, Gneveckow U et al (2006) Thermotherapy using magnetic nanoparticles combined with external radiation in an orthotopic rat model of prostate cancer. Prostate 66:97-104

32. Maier-Hauff K, Rothe R, Scholz R et al (2007) Intracranial thermotherapy using magnetic nanoparticles combined with external beam radiotherapy: results of a feasibility study on patients with glioblastoma multiforme. J Neurooncol 81:53-60

33. Johannsen M, Gneveckow U, Taymoorian K et al (2007) Morbidity and quality of life during thermotherapy using magnetic nanoparticles in locally recurrent prostate cancer: Results of a prospective phase I trial. Int J Hyperthermia 23:315-323

34. Johannsen M, Gneveckow U, Thiesen B et al (2007) Thermotherapy of prostate cancer using magnetic nanoparticles: feasibility, imaging, and three-dimensional temperature distribution. Eur Urol 52:1653-1662

35. van Landeghem FK, Maier-Hauff K, Jordan A et al (2009) Postmortem studies in glioblastoma patients treated with thermotherapy using magnetic nanoparticles. Biomaterials 30:52-57

36. Gneveckow U, Jordan A, Scholz R et al (2004) Description and characterization of the novel hyperthermia- and thermoablationsystem MFH 300F for clinical magnetic fluid hyperthermia. Med Phys 31:1444-1451

37. Scott CB, Scarantino C, Urtasun R et al (1998) Validation and predictive power of Radiation Therapy Oncology Group (RTOG) recursive partitioning analysis classes for malignant glioma patients: a report using RTOG 90-06. Int J Radiat Oncol Biol Phys 40:51-55 
38. Gorlia T, van den Bent MJ, Hegi ME et al (2008) Nomograms for predicting survival of patients with newly diagnosed glioblastoma: prognostic factor analysis of EORTC and NCIC trial 26981-22981/CE.3. Lancet Oncol 9:29-38

39. Wong ET, Hess KR, Gleason MJ et al (1999) Outcomes and prognostic factors in recurrent glioma patients enrolled onto phase II clinical trials. J Clin Oncol 17:2572-2578
40. Simon R (1986) Confidence intervals for reporting results of clinical trials. Ann Intern Med 105:429-435

41. Hadjipanayis CG, Machaidze R, Kaluzova M et al (2010) EGFRvIII antibody-conjugated iron oxide nanoparticles for magnetic resonance imaging-guided convection-enhanced delivery and targeted therapy of glioblastoma. Cancer Res 70:6303-6312 